\title{
CAUSALIDAD, PSEUDOCAUSALIDAD Y MEDICIÓN EN FÍSICA ${ }^{1}$
}

\author{
Wilfredo Quezada P. \\ Luis Pavez F. \\ Departamento de Filosofía \\ Universidad de Santiago de Chile \\ wiquezada@yahoo.com \\ pavezfloresluis@gmail.com
}

\begin{abstract}
Resumen
En esta ponencia nos proponemos mostrar que ciertas tesis o consensos recibidos sobre pseudoprocesos causales (PSP), procesos cuya característica prominente parece ser superar la velocidad de la luz, deben ser cuestionados o seriamente modificados. Dos de dichos consensos son, primero, que los PSP deberían ser tratados simplemente como "basura espacio-temporal" y, segundo, que ellos no cumplen, aparte de permitir aislar procesos causales genuinos, ninguna función epistémica o metodológica en la descripción de cualquier estado físico, en particular, al interior de la relatividad especial (RE). Para ello, apelaremos a procedimientos de medición física bien conocidos tanto en física clásica como en RE. Como consecuencia de lo anterior, argumentamos que, una vez que los PSP son integrados en toda la escena física, se revela que ellos juegan un rol fundamental y más complejo en la física experimental de lo que hasta ahora se ha pensado.
\end{abstract}

Palabras clave: procesos causales, pseudoprocesos, medición, relatividad especial y cantidades conservadas.

\section{Abstract}

In this paper we show that some received contentions about causal pseudo processes (PSP), processes of which the most salient aspect seems to be travelling faster than the speed of light, must be rejected o seriously modified. Two of such contentions are, first, that PSPs should be considered just "spatiotemporal junk" and, second, that they do not meet, besides allowing us to isolate proper genuine causal processes, any other epistemic or methodological role in the description of a physical state of affair, in particular, in a special relativity (SR) setting. In order to do that, we are going to appeal to physical measure procedures, well known both in classic and relativistic physics. As a consequence of that, we contend that, once PSPs are integrated into the whole physical scenario, a fundamental and more complex role for them in experimental physics is revealed.

KEY WORDS: causal processes, pseudoprocesses, physical measurement, special relativity, conserved quantities.

1 Este trabajo se desarrolló en el contexto del Proyecto Dicyt-Usach 031053QP. 


\section{Introducción}

$\overline{\mathrm{R}}$ Una parte de las discusiones filosóficas contemporáneas sobre relatividad especial (RE) se ha centrado en el problema, planteado muchos años atrás por $\mathrm{H}$. Reichenbach (Reichenbach 1958), de distinguir de una manera adecuada entre un proceso relativista genuinamente causal y uno que no lo es. A este segundo tipo de proceso Reichenbach lo llamó inicialmente secuencia causal irreal, pero preferiremos, siguiendo a W. Salmon (Salmon 1984), llamarlo pseudoproceso (PSP). En una primera aproximación, los PSP, como indica Reichenbach, parecen definirse adecuadamente como aquellos procesos que parecen justamente violar la constancia de la velocidad de la luz ${ }^{2}$. Ejemplos típicos de ellos son la traza que generaría un rayo de luz láser enviado desde la tierra y que barre una zona de la superficie de la luna conforme se mueve el puntero láser en la tierra, o el punto de fuga en que se cruzan dos reglas súper lumínicas ${ }^{3}$. Ya que, por esta razón, parecen no trasmitir ninguna información física, no pueden ser considerados secuencias de entidades reales (no parecen interactuar con nada) y no pueden ser objeto, por la misma razón, de interés para los físicos practicantes trabajando en RE. Sin embargo, en principio parece no haber razones para negar que son procesos y, por tanto, habitantes de una ontología procesual ${ }^{4}$.Para Reichenbach, en particular, era fundamental distinguirlos no porque representaran alguna amenaza para la plausibilidad de la RE, sino porque su exclusión era parte de la tarea fundamental de explicar la dirección de la flecha del tiempo sobre bases termodinámicas, mostrando que solo los procesos causales genuinos pueden manifestar irreversibilidad macro estadística $^{5}$. De manera que, si se viola el orden temporal, no podríamos tener ya más orden causal.

A partir del trabajo seminal de Reichenbach se ha generado, por consiguiente, una importante discusión acerca de las condiciones de adecuación de cualquier criterio postulado que distinguiese entre PSP y procesos causales y que, a su vez, sirviese el propósito de caracterizar, en acuerdo con las teorías físicas hoy prevalecientes, el vinculo causal. Esto fue lo que el mismo Reichenbach pretendió al ofrecer su criterio de

2 Puede resultar sorprendente, pero la experiencia de los autores sugiere que tales procesos son mediana o escasamente reconocidos por los físicos practicantes.

Cf. Reichenbach 1958, pp. 147-149 y p. 204.

4 Desde luego, admitir una ontología procesual presupone que en una descripción ontológica alternativa de la realidad física en términos, por ejemplo, solamente de eventos o sucesos (aquella favorecida clásicamente por la interpretación de Minkowski de la RE), la formulación del problema de los PSP se deflaciona a una simple distinción entre secuencias físicas reales e irreales. Por consiguiente, en nuestra aproximación, basada en una ontología de procesos, se asume que el problema de los PSP no consiste simplemente en trazar tal distinción. Esta es, por lo demás, la postura mayoritaria de los autores trabajando actualmente en causalidad física. Para una defensa sistemática de la necesidad de modificar la ontología eventualista de base de la RE a una ontología de procesos, cf. Salmon 1984, p. 144.

Reichenbach 1956, caps. 2 y 3. 
transmisión de marca como la vía para caracterizar físicamente los procesos causales genuinos (Reichenbach 1956). La subsecuente discusión, liderada fundamentalmente por W. Salmon (Salmon 1984) y P. Dowe (Dowe 2000), ha llevado a abandonar finalmente el criterio original reichenbachiano y a sustituirlo por otro formulado por Dowe. El criterio de Dowe (o Dowe-Salmon) implica que un proceso (entendido como una línea de mundo en un diagrama de Minkowski) para ser causal, debe poseer alguna cantidad conservada, de acuerdo a como éstas son definidas en física. Este entonces es el criterio de cantidad conservada o $\mathrm{CCC}^{6}$. Mucho más importante, para nuestra argumentación aquí, que evaluar en particular las bondades o las limitaciones del CCC, será la cuestión acerca del valor per se de los PSP en la concepción de Dowe. En su opinión, una vez hecha la anterior distinción y asumido CCC como criterio para caracterizar procesos causales genuinos, los PSP deben ser descartados como basura espacio temporal, es decir, como basura física similar a los time (o space) wise gerrymander que los físicos relativistas descartan sin mayor discusión en cualquier descripción de un estado físico. En palabras de Dowe:

\footnotetext{
"Worms in spacetime that are not processes I call,...., 'spatiotemporal junk'. Thus a line on a spacetime diagram represents either a process or a piece of spatiotemporal junk, and a process is either a causal or a pseudo process. In a sense, what counts as an object is unimportant; any old gerrymandered thing qualifies (except timewise gerrymanders). In the case of a causal process, what matters is whether the object possesses the right type of quantity. A shadow, for example, is an object, but it does not possess the right type of conserved quantities; a shadow cannot possess energy or momentum. It has other properties, such as shape, velocity and position, but possesses no conserved quantities" (Dowe, P., 2000, p. 91).
}

De esta manera, en opinión de Dowe, los PSP deben dejar de ser parte de la ontología procesual y deberían ser expulsados de la escena física, por no jugar en ella ningún rol metodológico ni epistemológico fundamental.

De todo lo someramente dicho antes, es posible deducir, a nuestro juicio, los siguientes consensos entre los teóricos de causalidad física interesados en PSP:

a. Los PSP causalmente relevantes solo pueden ocurrir a condición de que se viole la velocidad de la luz (Reichenbach).

b. Los PSP sirven solo un propósito filosófico en la medida en que distinguirlos de los procesos causales y aceptar irreversibilidad termodinámica permite salvar la explicación de la flecha temporal (Reichenbach).

c. Los PSP son basura espacio-temporal (Salmon/Dowe).

d. Los PSP no sirven ningún propósito (ni epistemológico ni metodológico) en la descripción de un estado físico real porque no pueden interactuar con procesos físicos genuinos (todos los autores).

6 Cf. Quezada 2002, para una síntesis y examen más detallado. 
Lo que nos proponemos en este trabajo es, en primer lugar, mostrar, apelando a argumentación física y hallazgos experimentales, que los consensos $a$ y $b$ deben ser modificados y que los consensos $c$ y $d$ son, tal como han sido formulados, errados $\mathrm{y}$ deberían ser abandonados. Para ello mostraremos que, contra la visión recibida sobre PSP, ellos han jugado históricamente y juegan actualmente un papel no menor en la medición en ciertas áreas de la física y parecen, por ende, más entretejidos con la práctica de la física de lo que se podría imaginar. Sugeriremos adicionalmente que nuestro argumento aquí, en lugar de mostrar un defecto mayor en la concepción Salmon/Dowe, lleva a extenderla de una manera fructífera en una dirección metodológica probablemente descuidada hasta ahora. Finalmente, esperamos que algunas de las cuestiones que diremos aquí permitan disponer las bases de alguna explicación unificadora de muy diversas manifestaciones físicas que parecen involucrar PSP.

\section{Historia de la física e ilusiones}

Una forma natural de admitir que somos víctimas constantemente de ilusiones físicas proviene de experiencias con sombras. Todos parecemos reconocer que, aunque ellas se basan en entidades físicas, la información que nos pueden entregar de estas últimas no es confiable. Sin embargo, reconocemos también que nos sentimos autorizados a hablar de ellas como si tuvieran alguna forma de existencia en el espacio tiempo. En esto justamente consiste su poder ilusorio. Con razón entonces las podemos llamar, de manera general por el momento, pseudoentidades físicas. Sin embargo, el reino de las pseudoentidades va mucho más allá del reino de las sombras. Piénsese, por ejemplo, en la señal luminosa proyectada perimetralmente por un faro y observada por un marino desde la distancia una noche neblinosa. Es obvio que, en este caso, diríamos, contra lo que pensaríamos normalmente de las sombras, que la ilusión creada en el marino es algo en lo cual él debería confiar ciegamente. Pero los ejemplos de pseudoentidades cotidianas no se limitan en lo absoluto a casos en los que la luz, o la ausencia de ella, juegue un papel privilegiado. Descubrimos muchos de ellos sobre todo cuando atendemos a fenómenos que se despliegan en el tiempo articulando una forma de proceso, aunque manteniendo cierta persistencia. Piénsese, por ejemplo, en el llamado efecto de la "ola", al que una muchedumbre en un estadio puede dar origen decidiendo espontáneamente ponerse de pie de manera secuenciada y sincronizada. Lo que parecemos observar entonces es un movimiento desplegándose en una determinada dirección y que parece obedecer a una cierta dinámica. Otro caso simple de un proceso ilusorio que involucra luz es la traza aparentemente continua de un rayo láser cuyo punto luminoso de interacción con la pared se mueve en ella con una cierta velocidad razonable ${ }^{7}$. Normalmente, la ilusión recae en que todos diríamos que ese rayo trazado en la pared es una entidad continua con una

Aun cuando el punto de luz no se mueva en la pared, este estará constituyendo un proceso, ya que muestra permanencia de estructura en el tiempo. 
breve existencia en el espacio tiempo. Finalmente encontramos procesos físicos ilusorios de base mecánica. Seguramente podríamos encontrar un buen análogo del ejemplo de la luz láser en el uso de un potente chorro de agua o pintura proyectado rápidamente en una pared. $\mathrm{O}$ en el barrido del disparo de una ametralladora a la misma velocidad. Todo lo que se obtenga en la pared es el resultado de un proceso cuya continuidad e identidad es, aunque a veces no lo creamos en el caso del agua o la pintura, ilusoria en la dirección que el agua, la pintura o la bala se mueven en la pared. Si quisiéramos aunar todos estos procesos con algún nombre particular, obviamente parecería correcto rotularlos como pseudoprocesos físicos, esto es, como nuestros PSP. Además, sería conveniente reunir en la misma categoría de los PSP a las pseudoentidades, pues no es difícil, al menos en lo que respecta a las sombras, mostrar que ellas se comportan como PSP, bajo una cierta definición de estos últimos.

En una primera aproximación, podríamos decir que, ya que los PSP involucran genuinas ilusiones físicas, es del todo esperable que las ciencias físicas no muestren interés alguno por ellos. Sin embargo, declarar esto ciertamente sería incurrir en un serio error histórico. Desde las aplicaciones más tempranas de la física se sabe que ella ha apelado a pseudoentidades, en particular, sombras, para determinar diversas mediciones del entorno físico. Por ejemplo es bien conocido que Eratóstenes utilizó la proyección de la sombra de una vara situada en la ciudad de Alejandría en relación con la completa iluminación de otra vara situada en la ciudad de Syene para determinar la magnitud del radio terrestre y, con ello, medir indirectamente la circunferencia de la Tierra ${ }^{8}$. De igual manera, sabemos que las antiguas civilizaciones medían el transcurso del tiempo a través de proyecciones de la sombra del sol. Por ejemplo, el astrónomo babilonio Berosus, en el siglo tercero A.C., construyó un reloj de sol hemisférico hecho de madera o piedra el cual consistía en una pieza cúbica con una cavidad hemisférica en la que descansaba una vara; la sombra de la vara proyectaba un arco cuya posición variaba con las estaciones ${ }^{9}$. En la parte interna se trazaban una serie de arcos, correspondientes a dichas estaciones, que, a su vez, se dividían en doce partes iguales, correspondientes a las doce horas de sol. La siguiente fotografía ilustra este tipo de reloj de sol.

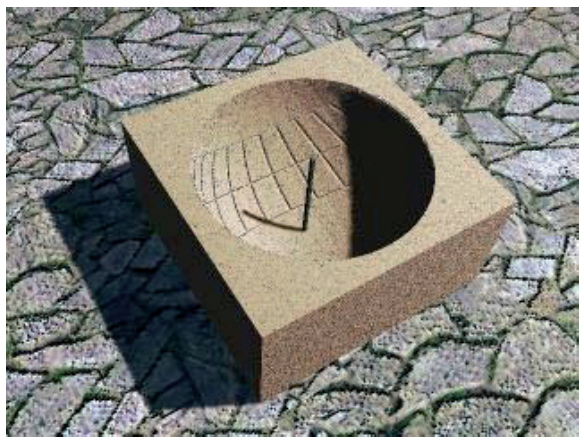

Figura 1

Para más detalles, véase Torretti 2007, pp. 83-85.

Véase, Valencia 2004, p. 4. 
Por otro lado, Aristarco de Samos en siglo III, A.C., utilizando luz y sombra, estimó la razón entre los tamaños de la Tierra, la Luna y el Sol y entre las distancias entre nuestro planeta y dichos astros. Aunque sus estimaciones no fueron suficientemente exactas, estaba empleando un método que al alcanzar mayor precisión resultaría muy eficiente. Torretti lo expresa de la siguiente manera:

"Cuando la Luna L, vista desde la Tierra T, aparece dividida en dos mitades, una oscura y la otra iluminada por el Sol S, las rectas LT y LS forman un ángulo recto. Un observador colocado sobre la Tierra puede medir el ángulo $\alpha$ entre la recta TL en dirección de la Luna y la recta TS en dirección del Sol” (Torretti, R., 2007, p. 98).

La siguiente figura ilustra a su vez esta descripción:

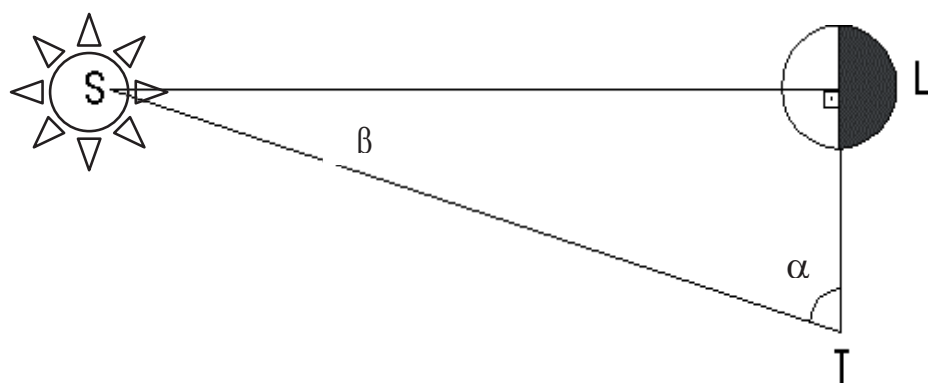

Figura 2

Es decir, a partir de la medida del ángulo $\alpha$ se puede establecer la relación entre la distancia que hay entre la Tierra y la Luna y la Tierra y el Sol. Aristarco estimó que la distancia al Sol es del orden de 19 veces la distancia a la Luna.

Como éstas, encontramos muchas otras situaciones en las que los físicos han apelado a proyecciones geométricas de sombras y haces lumínicos para determinar longitudes asociadas a cuerpos o volúmenes. Esto muestra obviamente que las sombras han sido desde la antigüedad una herramienta fundamental para llevar a cabo mediciones empíricas con valiosas aplicaciones practicas.

\section{PSP en física experimental clásica}

Más allá de los testimonios históricos referidos en la sección anterior, se puede mostrar que los PSP están íntimamente vinculados con la física experimental clásica, en particular, con la mecánica y la dinámica clásicas, en la medida en que muchas mediciones en aquellas áreas presuponen procedimientos pseudoprocesuales. Estos, nosotros creemos, son hechos tácitamente reconocidos en la práctica de la física experimental clásica, aunque inadecuadamente explicitados desde la perspectiva metodológica. Ilustraremos aquí dos aplicaciones normales que involucran PSP: 
a) Un PSP mecánico que mide un proceso mecánico

Un instrumento mecánico muy antiguo para medir fenómenos cinemáticos en el laboratorio físico es el timer. Este instrumento consiste en una cinta larga donde un brazo metálico con una pequeña terminación esférica en la punta golpea el paso de la cinta, dejándola grabada a través de una circunferencia de papel calco. Cada golpe que recibe la cinta (un tic) permite elaborar una unidad de tiempo (un tac) consistente en dos tics. Como se muestra en el diagrama siguiente, cualquier objeto que se mueva, ligado a la cinta, dejará su huella en los puntos discontinuos que genera su movimiento. Si el movimiento es con velocidad constante, el papel quedará con puntos separados por la misma distancia. El instrumento descrito cuenta con dos procesos mecánicos causales genuinos: (a) el vibrador y (b) el sistema mecánico cuya velocidad buscamos medir; y por otra parte, (c) un proceso no genuino o PSP, esto es, la secuencia de puntos sobre la cinta. Las unidades denominadas tics quedan completamente determinadas por la magnitud de la frecuencia del oscilador que mueve el brazo. Mientras mayor sea la frecuencia del brazo oscilante, más cercanos serán los puntos entre sí.

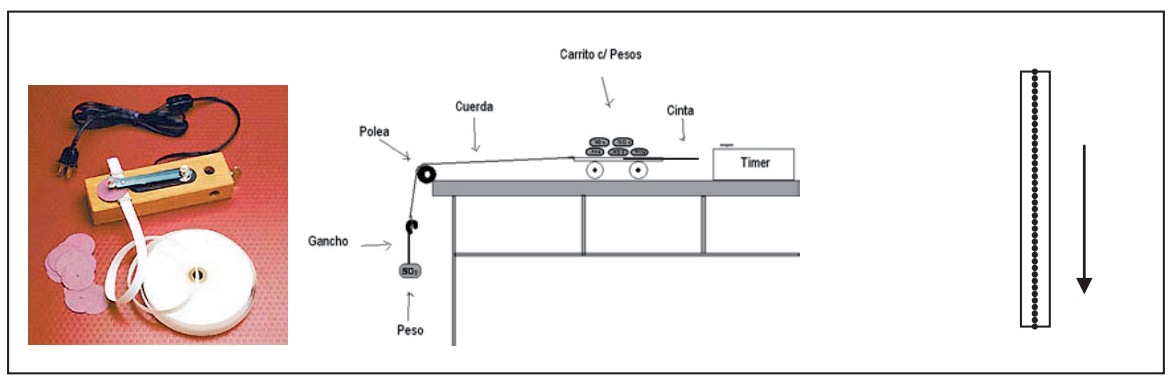

Figura 3

Se puede ver, de acuerdo a la imagen de la cinta, que la secuencia de puntos marcados en ella corresponde a un PSP discontinuo ${ }^{10}$. Sin embargo, este PSP representa la manera en que se movió el proceso físico continuo (el carro). Es decir, debido a la regularidad y continuidad del proceso que gesta al PSP, se puede inferir que entre punto y punto también se movió el carro, dada su persistencia como proceso. Los puntos marcados tienen un movimiento relativo a la cinta, en la dirección del movimiento, pero es un movimiento de un PSP que superviene de un proceso causal en la dirección dada.

Para determinar la velocidad del proceso, bastará entonces con saber solo dos datos: la frecuencia del timer, lo que permitirá determinar el tiempo entre punto y punto, y el largo de la cinta, que se obtiene con una regla graduada. De este modo, podemos medir la velocidad de un proceso mecánico a través de un PSP mecánico.

10 Como veremos más adelante, la continuidad o discontinuidad no es una característica definidora de los PSP. 
b) Un PSP óptico que mide un proceso mecánico

Otro procedimiento de medición interesante que supone la apelación a PSP es uno que se usa comúnmente para inferir la velocidad angular de nuestro planeta en relación con su eje. Este procedimiento consiste en hacer pasar un flujo de luz a través de un agujero hecho en la cubierta de un material opaco, que, por ejemplo, puede ser la cubierta de una mesa plástica con un orificio pequeño en su centro. A continuación se dispone que la luz solar atraviese el orificio de manera que alcance el suelo, sobre cuya superficie hemos fijado un papel blanco. Si dejamos transcurrir un lapso de tiempo razonable, el punto de luz se desplazará de manera aparentemente continua en la superficie del papel, generando una lenta pero clara trayectoria. Por ejemplo, sí el lapso de tiempo toma 10 minutos, aproximadamente, se podrá observar un lento desplazamiento del punto luminoso del orden de unos $4 \mathrm{~cm}$. Este fenómeno de movimiento permite evidenciar que el sol se mueve respecto a la superficie de la tierra, o de manera inversa, que la tierra gira sobre su eje respecto al sol, que se supone fijo. La siguiente figura muestra el montaje experimental en el centro y tanto a la izquierda como a la derecha el punto de luz desplazado los $4 \mathrm{~cm}$.

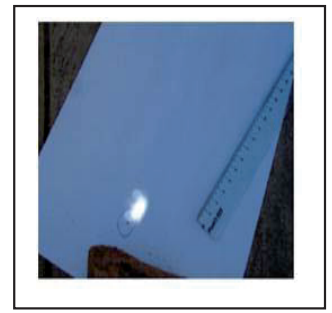

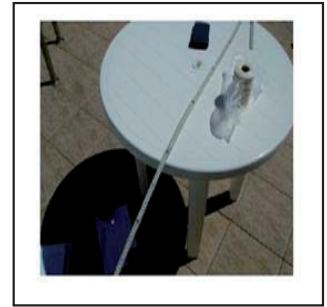

Figura 4



En estas condiciones, bastará entonces con utilizar el tiempo medido (10 minutos), el desplazamiento del punto de luz en el papel (h), la distancia desde el agujero hasta la marca de luz (d) y la distancia que separa al sol de la tierra (D, que corresponde a $1 \mathrm{U}-\mathrm{A})$, para inferir a través de una simple relación geométrica, que se ilustra en la figura siguiente, la velocidad angular de nuestro planeta $(\omega=\mathrm{V} / \mathrm{D}=\mathrm{H} / \mathrm{tD})$, cuyo valor es del orden de $7 \times 10^{-5} \mathrm{rad} / \mathrm{s}$.

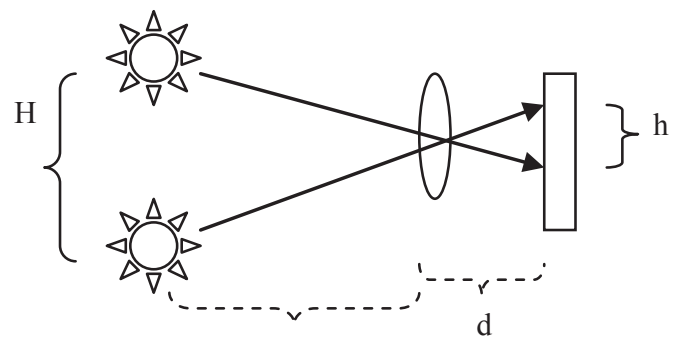

$\mathrm{D}$

Figura 5 
Es decir, hemos medido una magnitud mecánica (cinemática) a través de un PSP lumínico o electromagnético.

\section{PSP: su utilidad en procesos de medición en RE}

Como hemos indicado en la sección anterior, la apelación a PSP en la práctica de la física experimental clásica es un rasgo que parece metodológicamente irrenunciable, en la medida en que muchos de sus procedimientos de medición los presuponen de manera esencial (como asimismo, e indirectamente, la misma enseñanza de la física en el laboratorio). Sin embargo, en el terreno de la física contemporánea, en particular, en el ámbito de la teoría de la relatividad especial (RE), el asunto puede ser muy diferente. Desde el desarrollo de las concepciones de causación física a partir del trabajo de Reichenbach (Reichenbach 1958, 1956), de W. Salmon (Salmon 1984), basado en la idea de proceso causal, y su más reciente reformulación en manos de P. Dowe (Dowe 2000), basado a su vez en la idea de posesión de cantidades conservadas, se ha establecido entre los defensores de la causación física en RE la idea de que, ya que los PSP relevantes en RE pueden violar siempre la velocidad de la luz c, una vez que son adecuadamente distinguidos de los procesos causales genuinos, no deberían jugar ningún rol significativo en la descripción de un estado físico. Es lo que hemos ilustrado anteriormente con la cita de las palabras de Dowe en la introducción de este trabajo. La razón de fondo es que los PSP relacionan eventos que están causalmente desconectados, lo que implica que la dirección de la flecha temporal se puede invertir y, por lo tanto, no tienen la capacidad de interactuar con ningún proceso físico genuino. Consecuentemente, y como ya indicamos, Dowe, siguiendo a Salmon, los ha catalogado de "spatiotemporal junk" y ha sugerido desterrarlos de la escena física. Por lo tanto, si, como hemos venido sosteniendo, los PSP parecen ser fundamentales a la hora de obtener información esencial acerca de todo tipo de proceso físico genuino, deberemos ahora argumentar a favor del valor de ellos al interior de ciertos procesos estudiados en RE, y con ello, desafiar en este punto específico la opinión de los mencionados teóricos de causación física.

En esta ocasión nos concentraremos en procesos de altas energías, en particular, procesos que implican chorros de partículas de centros galácticos activos (AGN). Es evidente que no podemos interactuar directamente con este tipo de procesos, por dos simples razones: en primer lugar porque se encuentran muy distantes de nuestro planeta $\mathrm{y}$, en segundo lugar, porque, aunque pudiésemos acercarnos lo suficiente, los altos niveles de energía que poseen impedirían físicamente nuestro trabajo. Sin embargo, la cosmología contemporánea ha encontrado maneras indirectas de capturar la información física relevante que proviene de dichos centros galácticos, focalizándose en la radiación electromagnética que emiten al espacio en todas las direcciones, como consecuencia de los procesos físicos que ocurren en su interior. Mostraremos en lo siguiente que los más interesantes procedimientos de medición indirecta de dicha radiación se llevan a cabo apelando a alguna forma de PSP electromagnéticos, ya sea a velocidades menores que 
la rapidez de la luz o mayor que ella, y que es esto lo que los hace procesos susceptibles de ser estudiados apelando a RE.

Un ejemplo dramático de dicha medición psudoprocesual en procesos cosmológicos de altas velocidades que se generan en AGN son aquellos que presuponen la supuesta presencia de agujeros negros súper masivos en su interior. Un ejemplo de este tipo reportado recientemente corresponde a la radiogalaxia tipificada con la serie 3C120. Dicha galaxia tiene la particularidad de enviar un chorro de materia lumínica radiante con un ángulo pequeño, respecto a la línea de visión terrestre, y con una gran velocidad, generando con ello un PSP óptico súper lumínico en la zona del laboratorio terrestre. En un artículo publicado por Gómez y Steffen, el año 2009, se da cuenta sobre esta medición en los siguientes términos:

"Las regiones de emisión más intensa, o "componentes", del chorro muestran un comportamiento característico de estas fuentes, pero no por ello menos intrigante: su movimiento proyectado en el plano del cielo es mucho más rápido que la luz. La primera vez que se observó el movimiento súper lumínico, en los años setenta, se pensó que violaba la teoría de la relatividad de Einstein. Sin embargo, es perfectamente explicable dentro de la teoría de la relatividad, y nos ha permitido constatar un hecho de suma importancia en el estudio de los chorros en AGN: el material que viaja a lo largo de los chorros ha de hacerlo a una velocidad que, si bien no es superior a la velocidad de la luz, ha de hallarse muy cercana a la misma (del orden del $90 \%$ o superior); además, ha de viajar en una dirección cercana a la de la visual " (Gómez y Steffen 2009, p. 36).

La siguiente imagen muestra este tipo de componentes.


Figura 6

En este sistema de genuinos procesos causales y PSP deben considerarse tres tipos de procesos: el primero corresponde al chorro o componente, que es un auténtico proceso causal que se desplaza a una rapidez de $0.9 \mathrm{C}$; el segundo consiste en la información electromagnética o radiación que se desplaza en el vacío con velocidad $C=3 \times 10^{8} \mathrm{~m} / \mathrm{s}$, que también es un proceso causal y es el conjunto de procesos que arriban a nuestro planeta; y el tercero es el PSP compuesto de las distintas interacciones entre la información electromagnética o radiación y el instrumento de captación en nuestro planeta. 
A continuación exponemos visualmente el esquema del chorro y la forma en que se detecta desde nuestro planeta la radiación enviada por este, y que permitirá formar en los detectores terrestres el PSP medido. La imagen ilustra, además, el vector velocidad del chorro (flecha blanca diagonal), que alcanza una velocidad cuya magnitud es casi tan grande como la rapidez de la luz en el vacío ( $0.9 \mathrm{C}$, como ya dijimos). Con color blanco, pero hacia abajo de la figura, se observa el vector que representa la velocidad de la luz en dirección hacia nosotros. Para el chorro de la radio galaxia 3C120, la angulación del chorro respecto a la dirección en que viaja la luz hasta nuestro planeta corresponde aproximadamente a $20^{\circ}$.

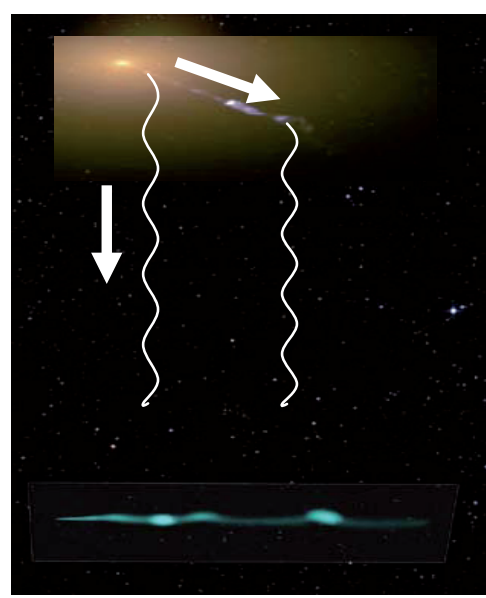

Figura 7

A continuación exponemos el esquema de medición de los procesos involucrados. Según se aprecia en la imagen anterior, los tres tipos de procesos ocurren en el plano $\mathrm{X}-\mathrm{Y}$. El desplazamiento del chorro tendrá entonces una componente en el eje Y, dada por $\mathrm{V} \Delta \mathrm{t} \cos \theta$ y otra en el eje $\mathrm{X}$, dada por $\mathrm{V} \Delta \mathrm{t} \operatorname{sen} \theta$. Luego, habrá tres velocidades a relacionar: la velocidad del chorro, V, la velocidad de la radiación, C, y la velocidad del PSP, que identificaremos con el símbolo $\mathrm{V}_{\mathrm{PSP}}$. 




Figura 8

Aplicando algunos teoremas básicos a los elementos geométricos del esquema esbozado y considerando los principios de la RE, se puede derivar fácilmente una relación entre la velocidad del chorro ${ }^{11}$, V, la velocidad de la luz, C, y la velocidad del PSP que se genera en el plano de recepción, $\mathrm{V}_{\mathrm{PSP}}$. La mencionada relación es la siguiente:

$$
V_{P S P}=\frac{V \operatorname{sen} \theta}{1-\frac{V}{C} \cos \theta}
$$

Si procedemos a analizar la relación dada podemos obtener los siguientes resultados:

(a) Si el ángulo del chorro es de $90^{\circ}, \operatorname{sen} 90^{\circ}=1 \mathrm{y} \cos 90^{\circ}=0$, entonces se obtiene que $V_{P S P}=V$ (velocidad del chorro). Es decir, la velocidad medida nos está informando que el chorro se mueve con la misma rapidez que el PSP y en la misma dirección.

(b) Si el ángulo del chorro es de $0^{\circ}, \operatorname{sen} 0^{\circ}=0 \mathrm{y} \cos 0^{\circ}=1$, entonces se obtiene que $V_{P S P}$ $=0$.

11 Para derivar la relación bastará con realizar una operación de diferencia y otra de cuociente. 
En este caso, el chorro o está en reposo o se mueve hacia nosotros, con respecto a nuestro planeta.

(c) Para el caso del chorro cosmológico, si tomamos un ángulo de $20^{\circ} \mathrm{y}$ una velocidad del chorro de $0.9 \mathrm{C}$, entonces la velocidad del PSP resultará de magnitud, $V_{P S P}$ $=2 C$.

Es decir, se nos presenta un PSP súper lumínico. Como ya indicamos anteriormente, fueron estos tipos de procesos los que llamaron la atención inicialmente a Hans Reichenbach.

(d) Si preguntamos ahora, ¿para qué velocidad del chorro, dado el ángulo de $20^{\circ}$, se produce un PSP con la misma velocidad que la velocidad de la luz? La respuesta deberá ser: $V=V_{\text {chorro }}=0,78 C$. Es decir, para cualquier velocidad menor que el $78 \%$ de la velocidad de la luz, el chorro genera PSP con rapidez menor que la luz en el vacío, y para velocidades del chorro superiores al 78\% de la velocidad de la luz se generan PSP súper lumínicos.

Este último resultado nos indica que, para chorros con rapidez menor que $0.78 \mathrm{C}$, los PSP generados tendrán que ser también más lentos que la luz. Una cuestión relevante en este contexto entonces es que PSP con rapidez menor que $\mathrm{C}$, como aquellos indicados en la sección 3 para la física clásica, también se pueden generar con procesos de altas energías estudiados en RE, y no llamarían la atención de físicos y filósofos más de lo que la han llamado los primeros. Es recién cuando los PSP superan la rapidez de la luz que se plantea, como hemos visto con el artículo de Gómez y Steffens, el llamado de atención. Sin embargo, y de acuerdo a lo que hemos venido mostrando en este trabajo, nada de esto es metodológica ni epistemológicamente problemático, ya que los PSP se pueden mover a cualquier velocidad, sin importar su naturaleza, y pueden ser medidos de diferentes maneras dependiendo de la teoría del caso.

Finalmente, se ilustra la situación ya descrita en un diagrama espacio tiempo de Minkowski en 2D(T,Y) y luego en 3D(T,X,Y

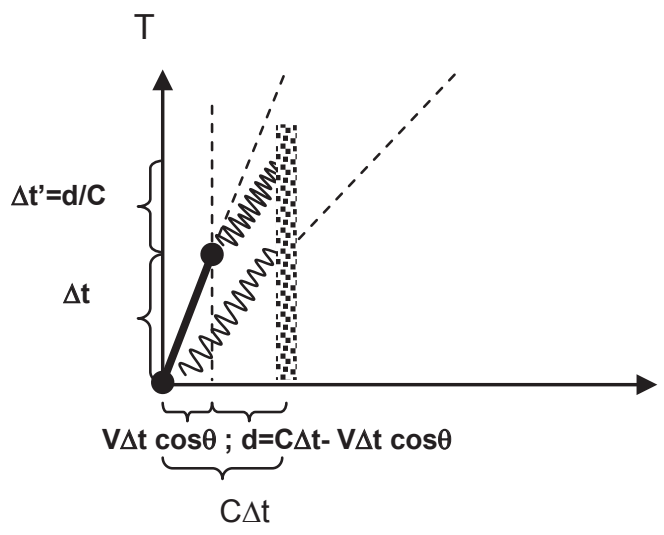




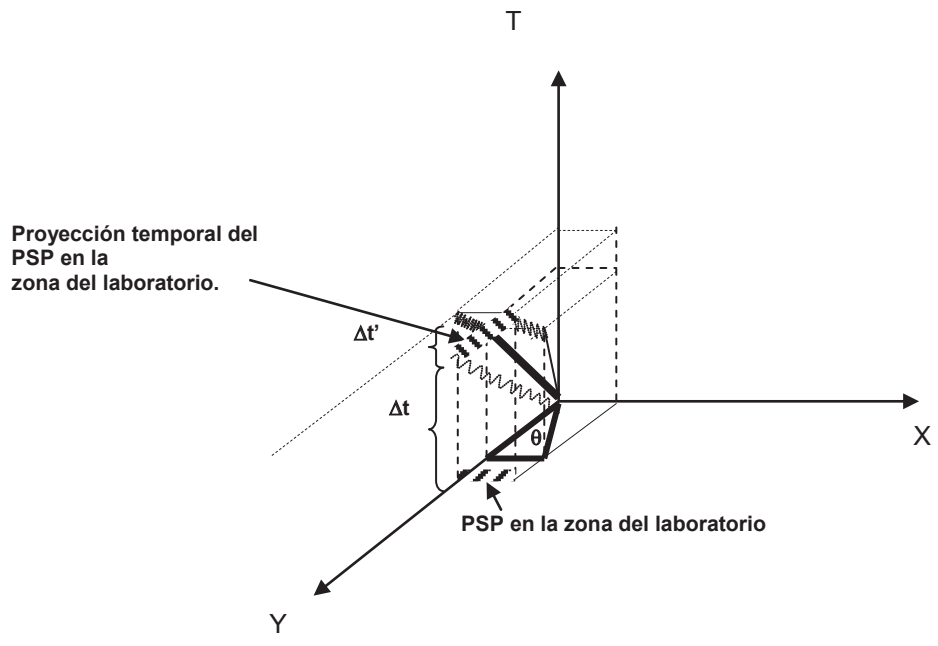

Figura 9

\section{PSP, espacialidad y medición}

El ejemplo analizado de medición pseudoprocesual en RE muestra, como en los casos clásicos, que aunque los PSP no transmiten per se cantidades conservadas ya que en la dirección del movimiento en que aparecen no producen interacción, sin embargo, se originan como una secuencia de interacciones de genuinos procesos causales en otras direcciones del espacio. Tenemos entonces una aparente paradoja: cuerdas de interacciones debido a procesos genuinos en una dirección, generan un proceso falaz y no genuino en otra dirección y estos procesos a su vez permiten obtener información esencial sobre los primeros sin violar ninguna restricción relativista.

Por otro lado, no se puede obviar que, en un análisis de todos los procesos analizados, tanto clásicos como relativistas, la característica que parece presuponerse cuando los PSP se convierten en mecanismos de medición es la interacción de las direcciones espaciales de los procesos involucrados. Esto es, los grados de libertad o las distintas direcciones espaciales en que se manifiesten las interacciones resultarán fundamentales, ya que en la mayoría de $\operatorname{los} \operatorname{casos}^{12}$, la aparición de un PSP en una dirección, acusará una causa común, de muchos procesos, en otra dirección ${ }^{13}$.

12 Es posible encontrar un PSP en la misma dirección espacial que el proceso genuino gestor. Esto ocurre, por ejemplo, con las ondas componentes de un grupo de ellas. La así llamada velocidad de fase de una onda individual puede superar la velocidad del grupo. Sin embargo, esta velocidad de fase, aunque supera la velocidad de la luz, no transmite información ni cantidades conservadas, ya que sigue siendo la velocidad de grupo la que las posee.

13 Hemos prescindido, en nuestra presente explicación, de cualquier compromiso conceptual respecto a la interpretación más pertinente de las cantidades conservadas mismas, ya sea en 
Junto con el presupuesto de la interacción de direccionalidad espacial, constatamos un rasgo común a todos los procesos revisados hasta ahora: las propiedades (físicas) que se detectan punto a punto en el PSP son indistinguibles entre sí. Por ejemplo, para un PSP lumínico, podemos constatar fotón a fotón que cada uno de ellos posee la misma energía como propiedad, aun cuando sean partículas distintas pero indistinguibles. De la misma manera, podríamos, para otras propiedades físicas a ser medidas, constatar la misma indistinguibilidad. Es esta indistinguibilidad de propiedades lo que permite, a su vez, que los PSP se perciban normalmente como procesos regulares y persistentes.

Por otro lado, las propiedades que se capturan en el proceso causal de base mediante el proceso de medición apelando a PSP son del mismo tipo de aquellas que poseen estos últimos. Por ejemplo, en el caso relativista examinado estudiamos velocidades de PSP en función de velocidades de los procesos genuinos. Por lo tanto, en este caso, operamos midiendo sobre la base de una identidad de los tipos de propiedades involucradas en ambos procesos. Así, aunque es esencialmente correcto, como indican Salmon y Dowe, que los PSP no son entidades básicas y finalmente supervienen de procesos causales, creemos que podemos ahora dar una explicación más sustantiva de esta superveniencia: ella es posible porque las propiedades del PSP que se generan en la interacción espacial con un proceso genuino heredan su identidad de la identidad de las propiedades del proceso causal. Y es esto lo que los convierte directamente en elementos legítimamente observables que hablan indirectamente de conexiones causales que, en muchos casos, residen más allá de la observación, en lo que, bajo cierta lectura, llamaríamos el mundo real ${ }^{14}$.

Lo anterior lleva naturalmente a la cuestión de la medición. Anteriormente hemos mostrado cómo es posible medir, en diferentes dominios de la física, con PSP y, a partir de los resultados de tales mediciones, derivar propiedades de los procesos causales reales que los gestan. Tales mediciones no serían posibles, sin duda, sin asumir que los primeros interactúan (causalmente) con nuestro aparato perceptual y que, a partir de dicha interacción, inferimos cómo está estructurado causalmente el mundo en el dominio dado. Sin embargo, es evidente que tales mediciones responden, a su vez, a una elección de la teoría del caso para el dominio del caso. Esto indica que tales mediciones (y los resultados consiguientes obtenidos) son teórico-dependientes y, por ello, que los PSP siempre lo serán también. Estamos conscientes, sin embargo, de que ello, aunque sugiere una visión favorable a la concepción de carga-teórica-de-la observación, no debería considerarse como un argumento orientado a dar una plausibilidad especial a dicha tesis. Nosotros consideramos que en principio se deben tomar ciertas reservas

términos escalares o en términos vectoriales, debido a que dicha línea de análisis, aunque evidentemente relevante en una investigación completa y autocontenida del tema, no incide directamente en el objetivo de este trabajo.

14 Esta consecuencia para el nivel observacional o perceptual puede tener implicaciones no menores para otros ámbitos, por ejemplo, para el ámbito de la informática computacional y los softwares asociados, pues, al parecer, todas las animaciones computacionales operan sobre la base de estos supuestos. 
en lo relativo a lo que podemos concluir a este respecto. Esto debido al menos a dos razones que pasamos a examinar a continuación.

En primer lugar, gran parte de la argumentación desarrollada hasta ahora sugiere que lo no-causal superviene de lo causal o, en otras palabras, que lo último genera lo primero. Si se busca caracterizar esto de manera más clásica, uno podría ver esta relación en los siguientes términos. La medición (que obtenemos, en este caso, a través de nuestras percepciones de fenómenos pseudoprocesuales) revela información fundamental acerca de la estructura de los procesos causales genuinos y, por lo tanto, cumple obviamente una función esencialmente epistémica. Es esta entonces, podríamos pensar, la dimensión característica de los PSP y lo que, desde una perspectiva filosófica, hace toda la diferencia respecto a los procesos genuinos. Por otro lado, la estructura revelada de un proceso genuino provee el ingrediente ontológico en la explicación final del dominio físico estudiado. Así, la tesis de superveniencia implicaría que el ingrediente ontológico determina el contenido cognitivo. Como hemos visto, en un sentido específico esto es estrictamente correcto. Sin embargo, es posible mostrar también que la relación entre ambos niveles -lo ontológico y lo epistemológico- puede ser más compleja de lo que uno podría imaginar en este escenario. Esto significa que lo no-causal puede a lo menos revelar lo causal de una manera crucial o esencial. Un ejemplo paradigmático de esta relación peculiar se constata en la RE misma, la concepción física contemporánea con mayor importe causal que conozcamos y que ha servido de base para motivar filosóficamente las teorías de procesos causales.

Si apelamos de una manera simple a la manera en que podemos medir una vara en movimiento en RE, diremos que sus extremos A y B, que dan la extensión de la vara que se mueve respecto a un observador $\mathrm{S}$, son registrados simultáneamente por dicho observador. Esto para Einstein es fundamental, puesto que garantiza que cualquier par de entidades ubicadas en dichos puntos en el instante de medición quedan totalmente desconectados causalmente entre sí, garantizando la ausencia de influencia causal en la dirección del movimiento. También es sabido que si un segundo observador, digamos $\mathrm{S}^{\prime}$, se mueve con velocidad relativa a $\mathrm{S}$, en la misma dirección de la extensión de la vara medida, este nuevo observador medirá los eventos extremos de la vara de manera sucesiva, obteniendo, por ejemplo, el evento A antes que el evento B; sin embargo, un tercer observador que viaja también con velocidad relativa a $\mathrm{S}$, digamos $\mathrm{S}$ ", pero en el otro sentido del movimiento (distinto a $S$ ' respecto a $S$ ) medirá ambos eventos en un orden distinto a S', por ejemplo, registraría primero B y luego A. Lo interesante es que si existe al menos un observador que mide dos eventos separados espacialmente de manera simultánea, entonces cualquier medida sucesiva de dichos eventos tomada por cualquier otro observador en movimiento relativo al primero, mostrará una relación entre la distancia entre ellos y el tiempo medido, que supera la rapidez de la luz. Es decir, dichos eventos tendrán para todos los observadores una relación espacio-temporal así llamada de tipo espacio o espacialoide entre ellos.

De acuerdo a lo dicho y a la forma en que aquí se han explicado los PSP, podemos decir ahora que, en acuerdo con las recomendaciones metodológicas del mismo Einstein, una medición simultánea de dos puntos separados espacialmente en los extremos de una barra, se realiza fundamentalmente apelando a PSP súper lumínicos, 
en la dirección en que los puntos A y B se encuentran separados. En el caso de la medición del chorro súper lumínico, que hemos venido ejemplificando, bastaría con que un observador se moviera en la dirección del PSP súper lumínico, con una cierta velocidad relativa respecto al laboratorio acoplado a nuestro planeta, para poder medir simultáneamente los puntos extremos del PSP y, por ende, todos los puntos del PSP capturado y graficado ${ }^{15}$.

De lo anterior se sigue entonces que las prescripciones metodológicas para medición al interior de la RE, tal como fueron concebidas por Einstein, para cuerpos extensos desplazándose a altas velocidades, parecen presuponer eventos desconectados causalmente, esto es, PSP. Sin embargo, estas prescripciones sugieren, a su vez, que tales PSP son un mecanismo fundamental para revelar el comportamiento causal de objetos en movimiento relativo. Así, supuestos metodológicos e información asociados a entidades no-causales nos llevan a constataciones sobre la estructura causal del dominio estudiado. Por un lado, los procesos tanto mecánicos como electromagnéticos, no importando su dirección de movimiento, estarán dentro del dominio del cono de luz y, por lo tanto, representando el dominio causal; sin embargo, por otro lado, los PSP, al alcanzar cualquier rapidez, se tornan omniabarcadores de las relaciones espacio temporales del espacio-tiempo de Minkowski. Es decir, los PSP serán las únicas herramientas metodológicas que permiten explorar dicho dominio desde las más bajas velocidades, hasta velocidades súper lumínicas, sin perder su identidad.

Lo anterior entonces sugiere que las implicaciones filosóficas que se siguen de los PSP, tanto en la dimensión ontológica como epistemológica, deberían ser evaluadas con especial cuidado, en particular, en lo que respecta a la disputa realismo vs. antirrealismo.

\section{Evaluación final y prospectos}

Evaluamos en esta sección los resultados anteriores en relación con los consensos descritos en la sección 1. En primer lugar, el consenso $a$, que se derivaba de la concepción reichenbachiana original de los PSP, debería ser abandonado o reformulado inmediatamente, pues, al menos desde la perspectiva del quehacer de la física en general, tanto los PSP que rebasan la velocidad de la luz como los que no lo hacen, parecen igualmente valiosos para aislar y estudiar estructura causal en el mundo aunque, desde luego, unos y otros lo puedan ser por diferentes razones. Esto nos lleva al segundo consenso reichenbachiano. Las razones que invoca Reichenbach (y que también comparte Salmon, en línea con su realismo causal) para adjudicarle un valor relativo a los PSP son estrictamente funcionales a la persistencia de los procesos causales genuinos: su distinción de estos últimos permite salvar un rasgo fundamental del mundo, esto es, la asimetría de la flecha temporal. Sin embargo, los PSP a bajas

15 Recuérdese que la simultaneidad de eventos distantes es relativa en el marco de la RE. 
velocidades que se ocupan en la física clásica exhiben en nuestra opinión un valor filosófico igualmente indisputable: a través de la medición permiten explorar y determinar rasgos fundamentales de los procesos casuales genuinos y, con ello, proporcionan rutas epistémicas fundamentales para detectar estructura causal. Esta es entonces otra forma de interpretar la superveniencia de los PSP de procesos causales genuinos. En una palabra, el valor de los PSP reside en lo epistémico, en tanto que el de los procesos causales genuinos, en lo ontológico. Y esto podría verse como suficiente para obligar a una reformulación del segundo consenso reichenbachiano.

En lo que respecta al consenso $c$, tenemos ahora razones fundamentales y consistentes para rechazarlo sin mayor discusión, al menos desde una perspectiva filosófica. Esto nos lleva finalmente al último supuesto. Creemos que hemos mostrado también su insostenibilidad y, en contraste, la esencial e irrenunciable utilidad de los PSP en la física experimental contemporánea. Sin ellos, supuestos metodológicos fundamentales de ciertos procedimientos de medición y de la información que obtenemos, a partir de estos, sobre diferentes dominios físicos, parecerían perder una posibilidad genuina de explicación unificada.

Finalmente, todo lo que hemos dicho anteriormente no debería verse como una recomendación para explorar una nueva teoría de procesos causales, alternativa a aquella defendida por Dowe y Salmon, sino más bien como una propuesta de rectificación, refinamiento y, con ello, de extensión -seguramente inesperada- de la última.

\section{Referencias bibliográficas}

Dowe, P. (2000), Physical Causation. Cambridge: Cambridge University Press.

Gómez, J.M. y Steffen, W. (2009), “Agujeros negros supermasivos”, Investigación y ciencia. Vol. 396, p.36.

Quezada, W. (2002), “Causalidad física: procesos causales y cantidades conservadas", Revista de Filosofia, Universidad de Chile, Vol. LVIII.

Reichenbach, H. (1958), The Philosophy of Space and Time. New York: Dover.

Reichenbach, H. (1956), The Direction of Time. Berkeley: University of California Press; hay traducción española: Reichenbach, H. (1959), El Sentido del Tiempo. México: Universidad Nacional Autónoma de México.

Salmon, W. (1984), Scientific Explanation and the Causal Structure of the World. Princeton: Princeton University Press.

Torretti, R., (2007), De Eudoxo a Newton. Santiago: Ed. Universidad Diego Portales.

Valencia, A. (2004), De la técnica a la modernidad. Antioquía: Ed. Universidad de Antioquía. 